Journal of Engineering and Applied Sciences 14 (Special Issue 8): 10318-10324, 2019

ISSN: $1816-949 \mathrm{X}$

(C) Medwell Journals, 2019

\title{
M2M of Chicken Hatchery Base on Wireless Sensor Network and IOT
}

\author{
Hussain Salah Hassan, Laith A. Abdul-Rahaim and Ali Shaban Hassooni \\ Department of Electrical Engineering, University of Babylon, Babylon, Iraq
}

\begin{abstract}
In this research, design of M2M system using Wireless Sensor Networks (WSN) that measure and control environmental variables of an incubator and a hatcher and shows the environmental variables and controlled and set the limits of them on a 7" LCD display panel designed by 4D workshop and controlled by Arduino. The proposed system contains two control nodes to display and control on the environment of incubator and hatcher. Each control node consists of two different sensor types. Each sensor reading is a specific value of the environment. These sensors send these reading to the Arduino micro-controller card to analyze the reading and make actions to control on the machine while that these reading displayed on the touch screen. After the sensors reading displayed on the touch screen it will be sent by serial to the XBee shield that will send them to coordinator node. The coordinator will collect the readings from all nodes and then send these information using Wi-Fi shield to the remote web server. One part of system is fault diagnostic at the same time of monitoring of sensing nodes work. The system was capable to run standalone without computers to save the working hours of computer. The system also capable to save the history of environment variables in the server. The proposed system has the ability to work in two modes, automatic and manual mode to control on the machine. The hatchery manager can control the motors and heaters and solenoids directly by changing the system to manual mode. In the end, the system is able to send sensors data and the machine status over the internet and by using TCP/IP server to transfer the environmental information to the remote webpage and can be displayed anywhere in world.
\end{abstract}

$\underline{\text { Key words: Index terms-sensors WSN, ZigBee, LCD touch screen, 4D workshop, hatchery, environmental }}$

\section{INTRODUCTION}

With the development of society, the techniques used in agriculture must change to meet the needs of the people. The development of using Internet technology and Wireless SensorNetworks (WSN) has brought light to the development of agricultural modernization. Internet remote control of WSN used in agricultural has become the inevitable trend of agricultural informatization. As example of this application is using of the remote control and monitoring on a chicken hatchery with realized the precise measurement and real-time control of the incubator and hatcher.

The hatchery system consist of two machine 1 st is the incubator and the 2nd one is the hatcher. The monitoring system can implement the scientific management methods, improve crop, disaster prevention and increase production (Zhou et al., 2007). WSN is one of the most popular and widely used technology in last few years. The WSN consists from one or more sensor nodes with a radio device to establish wireless network. The WSN is the preferred method to achieve large works due to its low cost, efficiency and reliability. In this study, WSN was the heart of the proposed system. The WSN of the proposed system formatted from two nodes, named as: "coordinator node $\mathrm{CRN}$ and control nodes $\mathrm{CN}$
(Ahmed et al., 2015). The purpose of CNs is to measure environmental data of incubator and hatcher like temperature and humidity and ventilation. $\mathrm{CNs}$ numbers can be set and modified by the hatchery manager. The heat and the humidity of the machine environment will increase the importance of continuous monitoring and adjustment of environmental variables of incubators. In addition to CNs, the proposed system has LCD touch screen to monitor the system.

All applications of WSN was build depended on the following equation "Sensing $+\mathrm{CPU}+$ Radio $=$ Thousands of potential applications" (Ahmed et al., 2015; Xiao et al., 2015). The wireless sensor networks have many advantages to be interest fields in recent years (Guo and Zhang 2005). The architecture of WSN based on ZigBee including hardware and software parts. So, there are several standards can be used in as shown in Table 1 to understanding advantages and disadvantage of each technology and particular applications (Morais et al., 2006, Mohanarajah et al., 2015).

ZigBee is preferred to use in WSN compare with the other technology because ZigBee has "low data rate, low cost, long battery life, low power conception and also simple in used and has small size and little complexity" (Kehoe et al., 2015, Cebrat 2014).

Corresponding Author: Hussain Salah Hassan, Department of Electrical Engineering, University of Babylon, Babylon, Iraq 10318 
J. Eng. Applied Sci., 14 (Special Issue 8): 10318-10324, 2019

Table 1: Comparisons of different wireless standard technologies as taken from (Morais et al., 2006; Mohanarajah et al., 2015)

\begin{tabular}{llll}
\hline Features & Wi-Fi (IEEE802.11) & Bluetooth (IEEE802.15.1) & ZigBee (IEEE 802.15.4) \\
\hline Radio & DSSS & FHSS & DSSS \\
Data rate & $11 \mathrm{Mbps}$ & $1 \mathrm{Mbps}$ & $250 \mathrm{kbps}$ \\
Data type & Video, audio, graphics, files & Audio, graphics, picture, files & Small data packet \\
Range $(\mathrm{m})$ & 100 & 10 & 200 \\
Battery life & Hours & Weeks & Weeks to month \\
Power consumption & $160-600 \mathrm{~mW}$ & $40-100 \mathrm{~mW}$ & $2-63 \mathrm{~mW}$ \\
Cost $(\$)$ & 100 & 40 & $22-55$ \\
Complexity & complex & Very complex & Simple \\
\hline
\end{tabular}

Previous works: There are many previous works specialized in WSN and monitoring system in various areas of life. Ahmed et al. (2015) show how the application of ZigBee technology on the safe mine production. ZigBee device transmitted wirelessly the data that has been read by the sensors on the collier's body to the GWN that send them to the computer. Cao et al. (2006) presented ZigBee application in wireless natural gas counter log and transfer.

The development of implementation of ZigBee wireless network to control field bus system was present by Chen and Zhou (2006) which not only ensure the security and real-time communication but also reduced the cost of deployment and redeployment. Sarkimaki et al. (2006) presented applicability of ZigBee technology for measurements of the rotor electric motor. And it has discussed the requirements for data transmission, electrical structure and the provision of energy from the sensor also built torque sensor based on ZigBee wireless-Model test. Dan et al. (2015) presents the design and implementation of agriculture greenhouse environment monitoring system based on ZigBee technology, the wireless sensor and control nodes takes CC2530F 256 as core to control the environment data. Pasalic et al. (2015) presents the considered and described of possibilities and way to design and implement a ZigBee-based data transmission and monitoring wireless smart sensor network integrated with the internet. Abdul-Rahaim and Ali (2015) presents the design and implementation of large farm environment monitoring system based on WSN technology, the wireless sensor and control nodes takes many accurate sensors and built a communication link as core to remote control the environment data of many regions of farm. Ali and Amran (2016) proposed an egg incubator system for precision farming.

The disadvantage is that the temperature and humidity values can be monitored remotely in cloud but the values could only be controlled manually. Sruthi and Jayanthy (2017) proposed the development of cloud based incubator monitoring system using Raspberry Pi which used the Raspberry Pi to control the environment of the incubator remotely by using wireless technology and android phone.

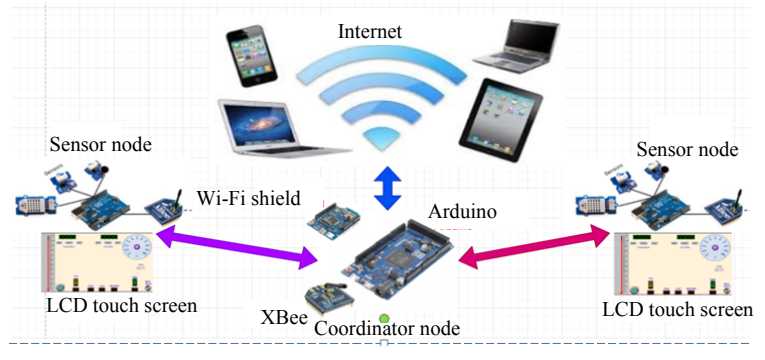

Fig. 1: System architecture of automation of chicken hatchery based on WSN and IoT

\section{MATERIALS AND METHODS}

The proposed WSN system has many nodes composed overall system for hatchery automation based on WSN and internet as shown in Fig. 1. Each node has two different type of modules sensor/motors module and the wireless module work at data rate of $9600 \mathrm{~b} \mathrm{sec}^{-1}$ with asynchronous wireless communication (Dan et al., 2015; Abdul-Rahaim and Ali, 2015). The proposed system's WSN follows IEEE 802.15.4 standard and routing protocol based on XBee (Abdul-Rahaim and Ali, 2015; Collier, 2015). The proposed system has two type of nodes. Each node takes its name and position due to its function in the system (Abdul-Rahaim and Ali, 2015; Shellshear et al., 2012).

The $\mathrm{CN}$ responsible for measuring "Temperature, humidity, ventilation fan, alarm system and the light" of the incubator as shown in Fig. 2. The $\mathrm{CN}$ is responsible on controlling the temperature and the humidity and switch the ventilation fan $\mathrm{On} / \mathrm{Off}$ and display the $\mathrm{CN}$ status on and the readings of the sensors on the 7" LCD touch screen panel. In $\mathrm{CN}$ the desired temperature and humidity of the incubator can be set by the same display panel. The CNs send all information of the $\mathrm{CN}$ to the CRN which is connected with Wi-Fi, so that, the information of all CNs will sent to the web server. The implementation of the proposed system was done by using ZigBee star topology for the WSN. The CNs set as ZigBee end device while $\mathrm{CRN}$ as ZigBee coordinator. In this work the $\mathrm{CN}$ has it's Arduino platform which can monitor and control on incubator or hatcher. The component of proposed system consists from following parts: 


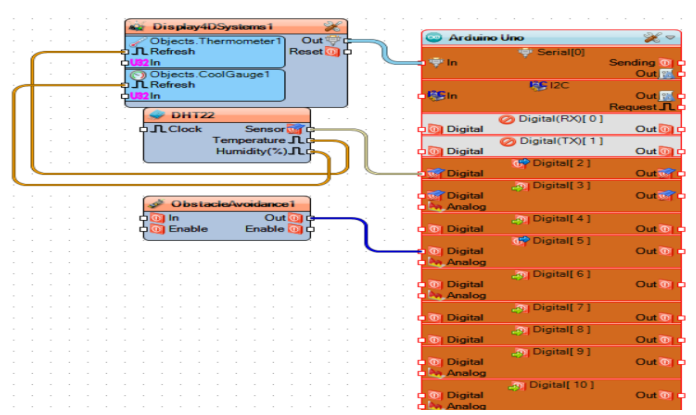

Fig. 2: Practical sensors connection to the Atmega328P diagram

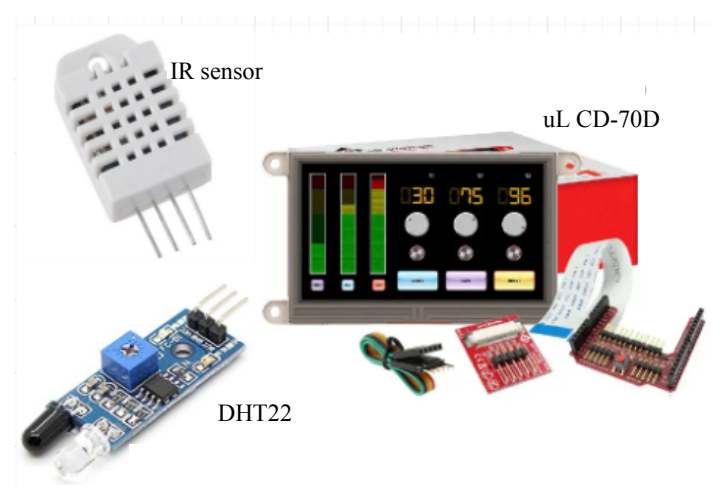

Fig. 3: DHT22, IR sensor and uLCD-70D

Design of control node $\mathrm{CN}$ : In this project the $\mathrm{CN}$ merged with the $\mathrm{SN}$ and contain Arduino platform which used to drive At-mega micro controller as shown in Fig. 2. The Arduino platform connected to all sensors and also connected to 7" LCD display touch screen as shown in Fig. 3. The Arduino platform contains everything needed to support the MCU include a clock circuit, voltage sources and USB serial driver to MCU. Highlevel language with compiler is used to programming the MCU. The "Integrated Development Environment" (IDE) is a program running on computer which allowed to draw sketches like block diagram or flow charts to program the Atmega328P controller. This software is an open source and based on $\mathrm{C} / \mathrm{C}++$ languages which then converted to machine language.

The CNs spends most of the time in sleep mode and it is still waiting to wake up from the CRN to perform one $\mathrm{jab}$ at a time. The CRN enables each sensor individually by using control signal then read data from the same sensor and processes these data and finally sends the processed data to transceiver through the serial connection $\mathrm{T}_{\mathrm{X}}, \mathrm{R}_{\mathrm{X}}$ (Shellshear et al., 2012, Riazuelo at al., 2015). The analogue to digital converter $\mathrm{ADC}$ that attached with each sensor converts the analog signal voltage to a 10-bit digital value using successive approximation technology which represent an integer between $0-1023$ where zero (a)

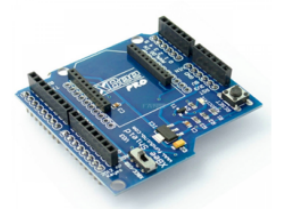

(b)

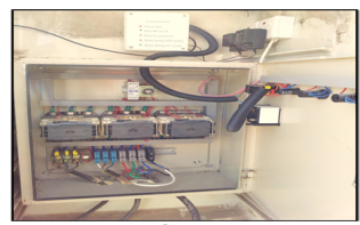

Fig. 4(a, b): Picture of the control node and drive to $3-\Phi$ motor

volt represent ground and most significant value 1023 represent $V_{\text {ref. }}$ The MCU manipulates sensors readings and send these readings to the display panel and to the $\mathrm{CRN}$ by the XBee which is connected to the $\mathrm{SN}$, then CRN send these reading wirelessly and serially through its Wi-Fi shield that to the web server to be then displayed by a designed web page throw the internet by using TCP/IP server protocol. The incubator SN used a temperature and humidity sensor DHT 22 to measure the environment status of the machine and use an IR sensor to check the belt of the ventilation fan for security. In general, the chicken hatchery consist of two machine, the first one is the hatcher and the second one is the incubator the required temperature for chicken egg incubation is 100 and the humidity level $90 \%$ for hatcher and the temperature is 99.5 and humidity about (85-87) for incubator (Boleli et al., 2016). The ventilation system of the incubator and the hatcher should be nonstop for $24 \mathrm{~h}$ to keep the embryos alive and do not choke.

The IR sensor used to ensure that the ventilation fan belt is ok and not broken. The temperature influenced the ability of grow the embryos. Therefore, knowledge of the temperature and humidity degree of incubators and hatchers will make it possible to give the right amount of heating or cooling to keep the embryos safe (Boleli et al., 2016). A capacitive humidity sensor and athermistor [DHT22] was used temperature sensors. Typically, this sensor can work and read the temperature between -40 to $80^{\circ} \mathrm{C}$ and humidity between $0-100 \%$.

Wireless comm unication system: XBee shield shown in Fig. 4 a, b used to connect Arduino platform with XBee module. XBee $63 \mathrm{~mW}$, from Digi International as wireless transceiver used to secure communication links between varies nodes of WSN. XBee module working with ZigBee protocol (Abdul-Rahaim and Ali, 2015; Kastner et al., 2014). The XBee modules provide a reliable low data rate delivery between two remote nodes.

Design of Coordinator Node (CRN): The CRN was erected on a place between the $\mathrm{SN}$ and the Wi-Fi router using a special drawer design which is responsible of connecting all the SNs with the web server CRN considered the brain of the system and all other nodes 


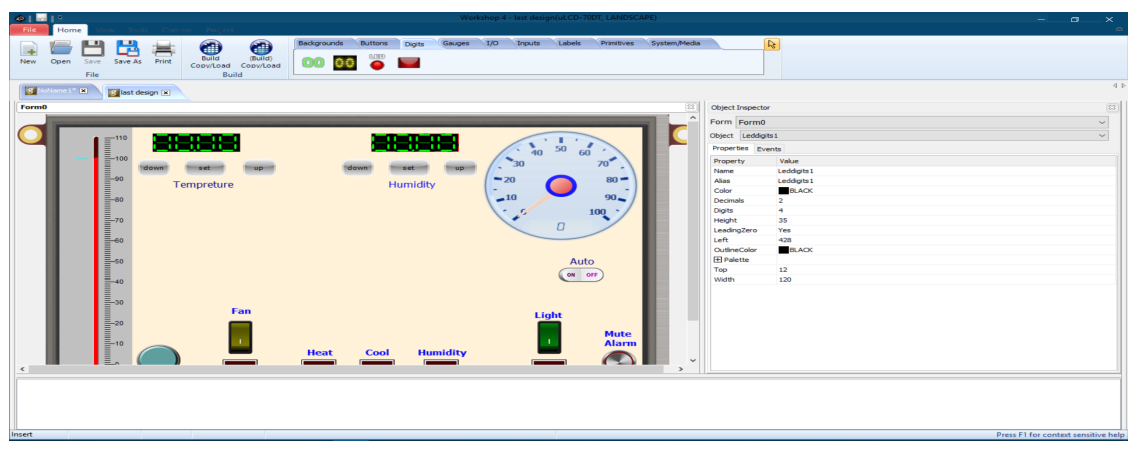

Fig. 5: 4D workshop to design the LCD screen

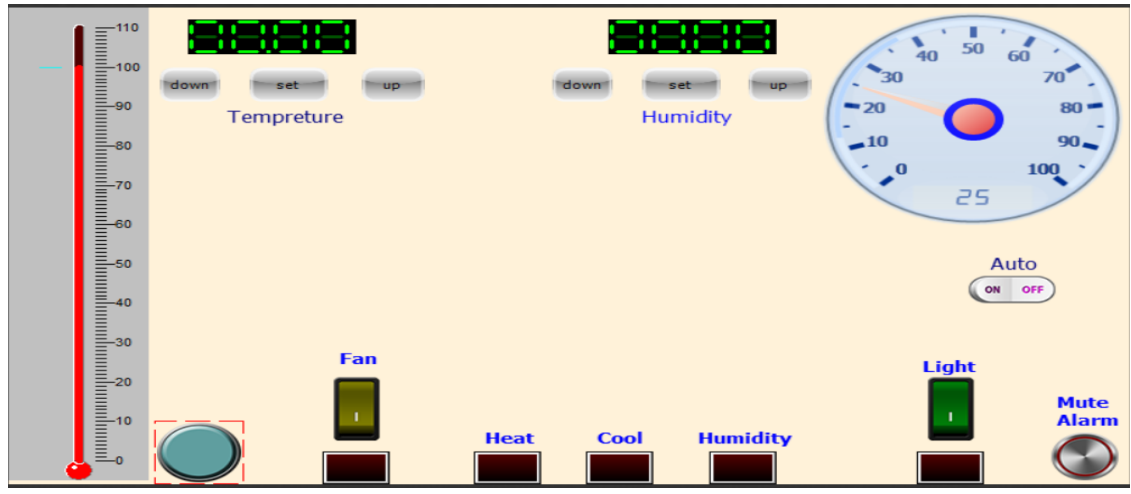

Fig. 6: Gages, buttoms, switches and LEDs form in LCD display for

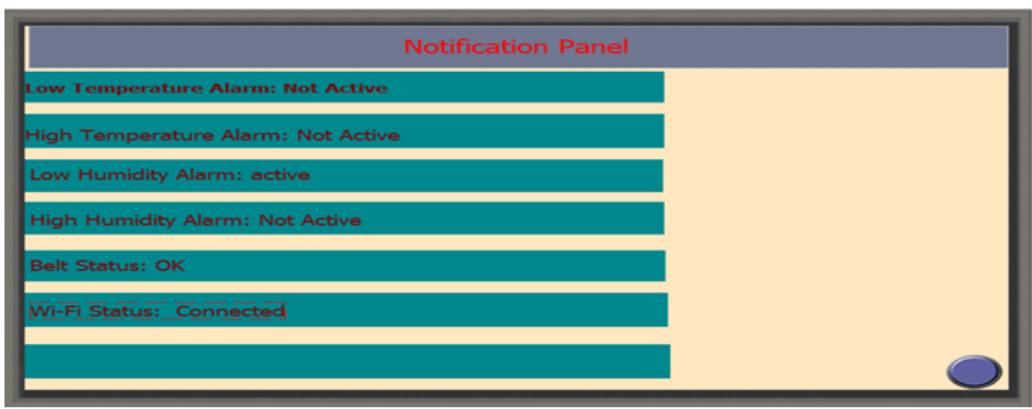

Fig. 7: Notification form in LCD display for NC

without the $\mathrm{CRN}$ will be inactive and will be waiting its permission to start sending its data to $\mathrm{CRN}$. The CRN has a unique 64 bit address. In this place sensing node combined with coordinator node. This node was established in the middle between sensing node and the Wi-Fi router. The design of this node divided into two parts, XBee configuration and MCU programming. CRN communicating with one end device node of WSN at any time.

4D System uLCD touch screen panel: The most important part of the proposed system is the LCD screen which used to display environmental sensor readings. The
4D workshop software has been used shown in Fig. 5 to create this user-friendly monitor interface which enables incubator and hatcher manager to monitoring and control the environment condition of incubator and hatcher and the status of WSN node, shown in Fig. 6 and 7. The LCD touch screen panel has many sectors or fields like, temperature and humidity parameters display, selecting buttons display, setting temperature and humidity controlling limits, system control mode switch, all that with on form, the other form display machine status and the alarm system of the machine which display the low and high temperature and humidity alarm, also display the belt status as shown in Fig. 6-8. The fist form of the 


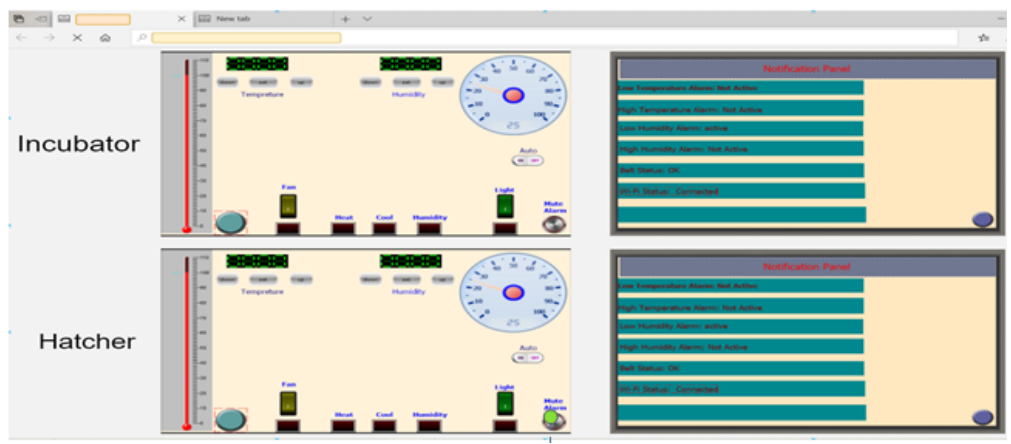

Fig. 8: Remote web page panel for two machine incubator and hatcher

panel consist of thermometer to display the real temperature value and a cool gage to display the humidity, also consist of two (four segments) LED digits to display the required temperature and humidity which represent the control value for the Arduino, also contain main button that select the machine working mode (automatic or manual) mode, also there is a fan and light button, alarm mute button. Also a button for form changing. The LCD display also has LED indicators for heating and cooling and light and contain two LED indicators to display actuator state.

Proposed system operation: The operation of the proposed system can be classified into the following parts to realize how the system work:

WSN system operation: All XBee transceiver of all nodes of WSN configured as End device ZigBee to connect with CRN only by adding the address of the coordinator in it packets shown in Fig. 1. The CRN has a unique address and a list of all nodes addresses stored in its SDRAM memory to use the addresses to communicate with each node of WSN. This way, given the hatchery manager the ability to add, remove or modify the numbers of nodes in the network to the desired limit and so, can add any new incubators or hatchers to proposed system. When the end device awake, it will send request messages to its $\mathrm{CRN}$.

The CRN when receives this request will check the packet queue to see if the program has any buffered messages for this end device. Then CRN send acknowledgment of MAC layer to the end device indicates if it has data to send to the end device or not. If the end device received the acknowledgment and find that CRN has no data for it, so, the device can eventually return to sleep or idle mode. Otherwise, it remains awake to receive the data. When $\mathrm{CRN}$ has received packages, begin to send each end device packet to the IP address throw Wi-Fi to the remote cloud using TCP/IP protocol, it allows the hatchery manager to monitor the end device status and also control on the end devices by changing the parameters that specify the end device work, all that by sending data packet form the web page of the cloud to the CRN. While CRN receives packages from each the web page, CRN will specify the packages address and send each packet to it's $\mathrm{CN}$.

The CN's MCU will receive a packet from the CRN and update its parameters like the specified temperature and humidity or change the system from auto to manual mode or vice versa. After that the $\mathrm{CN}$ will update it's display panel to show the data received from the CRN display plane. The system will begin a new cycle at the end of this step.

Surveillance and security system: The proposed system has been proposed surveillance locally and remotely the $\mathrm{SN}$ and monitoring the activity of the incubator and hatcher actuators and sensors and see real time of changing the temperature and the humidity and fluctuation between heating and cooling. Also the ventilation fan is also monitored and IR sensor will activate the alarm of the system if the motor belt cut. The security system will be in all $\mathrm{CNs}$, so, each $\mathrm{CN}$ will have a notification panel also connected to a buzzer to alarm the hatchery labor that there is something should be managed with the $\mathrm{CN}$. The buzzer will be activated in many cases, if the temperature or humidity get high or low to the set values or when the belt of the ventilation fan cut. This is a locally surveillance.

Since, all the $\mathrm{CN}$ connected to the $\mathrm{CRN}$ and the CRN connected to a web page using TCP/IP protocol over the internet, so that, all the data of the system will be display in the web page also the system can be controlled not only monitored in the web page. Also web page will display the history of the sensor reading of the $\mathrm{CN}$ during working time. Also the web page can save all the data received from the $\mathrm{CN}$ to be reviewed any time and also the remote panel can notify the hatchery manager if the alarm started in any node by sending notification massage. 
Internet remote access and automation: The designed web page panel has the ability to receive data from CRN through TCP/IP protocol via. the internet. So, the proposed system as mentioned above, used this feature to design two panels, each panel for a $\mathrm{CN}$ that can be used by the hatchery manger. The two panels are shown in Fig. 8. These web page panels can be used for $24 / 7$ access from remote computers or phones including connect/disconnect from the remote panel to access to the $\mathrm{CN}$ panel. The establishment of a connection between the remote computer or phone and $\mathrm{CN}$ through $\mathrm{CRN}$ needed the IP address of the web page.

The connection will be establishing after authorized and check the IP address of the remote panel. Each time run proposed automation web page panel, the system given a unique IP address which must be use these IP to establish communications link between to the remote computer or phone and CRN from any a place in world and watch and control the variations in the system in hatcher environmental variables by the hatchery manager as shown in Fig. 8.

\section{RESULTS AND DISCUSSION}

The proposed designed system in this study used to measure and control the environmental parameters of chicken hatchery, like "temperature, humidity, ventilation, heating and cooling". The obtained results, gives excellent results and control the hatchery environmental variables efficiently as shown in Fig. 7, 8 in incubator and hatcher machines and remote access computer everywhere in world. The proposed system had been tested for a period of two months in chicken hatcher in Babylon in Iraq and show that its response was stable; robust, save, high efficiency has precise reading with secure transferring of environmental information. Figure 7 and 8 illustrates incubator with live LCD touch screen display panel.

The proposed system will keep environmental variables in desired values between the two thresholds (max. and min.) which set by the hatchery manger. The proposed system keeps temperature between 99-100 and humidity between $85-90 \%$. The control of ventilation done by a fan connected to a rotational motor by belt to keep air as fresh as possible to ensure that the embryos will not junk. The incubator and hatcher temperature and humidity also has been measured and displayed on the LCD touch screen panel to let the hatchery manger knowing that the machine work probably and safe Fig. 8 shows temperature results for the incubator and hatcher.

\section{CONCLUSION}

The proposed designed system can monitor and control a lot of hatchery environmental condition values. The most important environmental variables that have been controlled such as temperature, humidity and ventilation. Temperature and humidity and good ventilation lead to get the best growth of the embryos in incubation or hachuring. The proposed system built based on WSN technology. Chicken's embryos are heavily dependent on the administration and therefore right adjustment of environmental conditions will give this yield. The proposed system uses sensors had high accuracy and fast response to read environment variables value, so, system reliability and life 'time' will be increase. The proposed system has friendly Graphical User Interface (GUI) designed by 4D workshop program and displayed on 7" LCD touch screen to display the environmental parameters and makes required decision when needed.

The proposed system can modify the total number of sensing nodes by added their address in SDRAM of Arduino platform of coordinator node to increase the coverage of the proposed system. The proposed system had error diagnostic subsystem working with the same time of monitoring. The proposed system also has the ability to save all data the proposed WSN system has the ability to run into standalone mode without computer to automate all the action to save hours of computer operation. The proposed system works in automatic mode as default system but the hatchery manger can change it to manual mode to control the motors and sensors directly. Finally, the best idea in the proposed system is the ability to connect it to the internet to transfer all the information to the hatchery manager anywhere in world. This operation of remote control is done by IP/TCP server protocol to exchange the information between incubators and hatchers $(\mathrm{CN})$ area and any remote computer or phone or tablet of the chicken hatchery manager.

\section{REFERENCES}

Abdul-Rahaim, L.A. and A.M.A. Ali, 2015. Remote wireless automation and monitoring of large farm using wireless sensors networks and internet. Int. J. Comput. Sci. Eng. Technol., 6: 118-137.

Ahmed, E., J. Qadir and A. Baig, 2015. High-throughput transmission-quality-aware broadcast routing in cognitive radio networks. Wirel. Netw., 21: 1193-1210.

Ali, F. and N.A. Amran, 2016. Development of an egg incubator using Raspberry Pi for precision farming. Int. J. Agric. For. Plantation, 2: 462-469.

Boleli, I.C., V.S. Morita, J.B. Matos Junior, M. Thimotheo and V.R. Almeida, 2016. Poultry egg incubation: Integrating and optimizing production efficiency. Braz. J. Poult. Sci., 18: 1-16.

Cao, Z., C.X. Cao and X.J. Tang, 2006. Design and application of wireless meter reading system based on ZigBee. Autom. Panorama, 23: 45-48.

Cebrat, G., 2014. Secure web based home automation: Application layer based security using embedded programmable logic controller. Proceedings of the 2014 2nd International Conference on Information and Communication Technology (ICoICT'14), May 28-30, 2014, IEEE, Bandung, Indonesia, pp: $302-307$. 
Chen, J.Y. and X.P. Zhou, 2006. ZigBee wireless communication technology in industrial controls. Radio Eng. China, 36: 61-64.

Collier, S.E., 2015. The emerging enernet: Convergence of the smart grid with the internet of things. Proceedings of the 2015 IEEE International Conference on Rural Electric Power (REPC), April 19-21, 2015, IEEE, Asheville, North Carolina, ISBN:978-1-4799-7555-6, pp: 65-68.

Dan, L., C. Xin, H. Chongwei and J. Liangliang, 2015. Intelligent agriculture greenhouse environment monitoring system based on IOT technology. Proceedings of the International Conference on Intelligent Transportation, Big Data and Smart City (ICITBS), December 19-20, 2015, IEEE, Halong Bay, Vietnam, ISBN:978-1-5090-0464-5, pp: 487-490.

Guo, L.S. and Q. Zhang, 2005. Wireless data fusion system for agricultural vehicle positioning. Biosyst. Eng., 91: 261-269.

Kastner, W., M. Kofler, M. Jung, G. Gridling and J. Weidinger, 2014. Building automation systems integration into the internet of things the IoT6 approach, its realization and validation. Proceedings of the 2014 IEEE International Conference on Emerging Technology and Factory Automation (ETFA'14), September 16-19, 2014, IEEE, Barcelona, Spain, pp: 1-9.

Kehoe, B., S. Patil, P. Abbeel and K. Goldberg, 2015. A survey of research on cloud robotics and automation. IEEE. Trans. Autom. Sci. Eng., 12: 398-409.

Mohanarajah, G., V. Usenko, M. Singh, R. D'Andrea and M. Waibel, 2015. Cloud-based collaborative 3D mapping in real-time with low-cost robots. IEEE. Trans. Automation Sci. Eng., 12: 423-431.

Morais, R., A. Valente, J.C. Almeida, A.M. Silva and S. Soares et al., 2006. Concept study of an implantable microsystem for electrical resistance and temperature measurements in dairy cows, suitable for estrus detection. Sens. Actuators A. Phys., 132: 354-361.
Pasalic, D., D. Bundalo, Z. Bundalo and B. Cvijic, 2015. ZigBee-based data transmission and monitoring wireless smart sensor network integrated with the Internet. Proceedings of the 2015 4th Mediterranean Conference on Embedded Computing (MECO'15), June 14-18, 2015, IEEE, Budva, Montenegro, pp: 240-243.

Riazuelo, L., M. Tenorth, D. di Marco, M. Salas and D. Galvez-Lopez et al., 2015. RoboEarth semantic mapping: A cloud enabled knowledge-based approach. IEEE. Trans. Autom. Sci. Eng., 12: 432-443.

Sarkimaki, V., R. Tiainen, T. Lindh and J. Ahola, 2006. Applicability of ZigBee technology to electric motor rotor measurements. Proceedings of the International Symposium on Power Electronics, Electrical Drives, Automation and Motion (SPEEDAM'06), May 23-26, 2006, IEEE, Taormina, Italy, pp: 137-141.

Shellshear, E., R. Berlin and J.S. Carlson, 2012. Maximizing smart factory systems by incrementally updating point clouds. IEEE. Comput. Graphics Appl., 35: 62-69.

Sruthi, B.M. and S. Jayanthy, 2017. Development of cloud based incubator monitoring system using raspberry Pi. Int. J. Edu. Manage. Eng., 7: 35-44.

Xiao, Z., X. Tan, X. Chen, S. Chen and Z. Zhang et al., 2015. An implantable RFID sensor tag toward continuous glucose monitoring. IEEE. J. Biomed. Health Inf., 19: 910-919.

Zhou, Y., X. Yang, X. Guo, M. Zhou and L. Wang, 2007. A design of greenhouse monitoring \& control system based on ZigBee wireless sensor network. Proceedings of the 2007 International Conference on Wireless Communications, Networking and Mobile Computing, September 21-25, 2007, IEEE, Shanghai, China, pp: 2563-2567. 